\title{
Ethylenediaminetetraacetic Acid-Assisted Synthesis of Nano Antimony Oxide by Microwave Method
}

\author{
Azadeh Tadjarodi*, Mohammad karimpour \\ Department of Chemistry, Iran University of Science and Technology, Narmak, \\ Tehran 16846-13114, Iran \\ E-mail: tajarodi@iust.ac.ir
}

\begin{abstract}
Organic ligands are widely used in the synthesis of metallic complexes, which can be burned and eliminated from the coordination sphere at high temperatures and remaining only oxide group. The reaction of Ethylenediaminetetraacetic acid (EDTA) as an organic ligand and antimony trichloride in a domestic microwave leads to formation of antimony-EDTA complex. Then, the resulting compound was calcined and got nano antimony oxide. The product was characterized by powder x-ray diffraction (XRD), fourier transform infrared spectroscopy (FT-IR), field emission scanning electron microscopy (FE-SEM) and UV-vis diffuse reflectance spectrum (DRS). This technique is a simple, fast, environmental friendly and can draw a usable viewpoint for the synthesis of nanomaterials.
\end{abstract}

Keywords: Microwave, Green, EDTA, Antimony oxide

\section{Introduction}

Oxide nanoparticles have attracted great attention over past decade due to novel properties compared with bulk materials $[1,2,3]$. There are many reports on the synthesis, structure, and applications of nanocrystalline materials [4,5,6,7]. Antimony oxides are well known to exist in several different phases including $\mathrm{Sb}_{2} \mathrm{O}_{3}, \mathrm{Sb}_{2} \mathrm{O}_{4}$, $\mathrm{Sb}_{6} \mathrm{O}_{13}, \mathrm{Sb}_{2} \mathrm{O}_{5}$ and the polymorphism, which have attracted great attention in the field of catalysts, medicines, optics and semiconductors. Among the different antimony 
oxides, $\mathrm{Sb}_{2} \mathrm{O}_{4}$ is the most common form of polymorphism, which has two types, namely the orthorhombic $\alpha$ phase and monoclinic $\beta$ phase, respectively [8]. $\alpha-\mathrm{Sb}_{2} \mathrm{O}_{4}$ has been widely used together with other oxides (e.g. $\mathrm{Fe}_{2} \mathrm{O}_{3}, \mathrm{MoO}_{3}$ etc.) as a catalyst for the ammoxidation of propane to acrylonitrile, the oxidation of propane or propene to acrylic acid and for hydrocarbon cracking and hydrogen reduction, and in the production of ceramic enamels $[9,10]$. In this paper, for the first time, we report the synthesis of leaf-like $\alpha-\mathrm{Sb}_{2} \mathrm{O}_{4}$ nanostructures by the microwave assisted method, which is a simple, fast, environmental friendly and green technique.

\section{Experimental}

\subsection{Materials of synthesis}

All of the chemical reagents used in this experiment were analytical grade and were used without further purification. Deionized water was used throughout.

\subsection{Preparation}

$912 \mathrm{mg}$ of antimony trichloride, $\mathrm{SbCl}_{3}$, dissolved in deionized water and stirred for 10 min. Then $744 \mathrm{mg}$ of EDTA under stirring was slowly added into this solutions, and stirred for $15 \mathrm{~min}$. The resultant emulsion transferred into a microwave system in $180 \mathrm{~W}$ for $20 \mathrm{~min}$. The resulting product was collected, filtered and washed with deionized water for several times to remove residual ions in the product, and drying at $80^{\circ} \mathrm{C}$ in air atmosphere for $3 \mathrm{~h}$ to produce the white products. Then the resulting powder was calcinated at $600{ }^{\circ} \mathrm{C}$ under the ambient atmosphere for $2 \mathrm{~h}$ and obtained white powder.

\subsection{Characterization}

The morphology of the product was observed directly by field emission scanning electron microscope (FE-SEM,VEGA/TESKAN microscope with an accelerating voltage of $30.00 \mathrm{KV}$ ). The phase of the product was determined by powder X-ray diffraction (XRD) analysis on a JEOL X-ray diffractometer with monochromatized $\mathrm{Cu}$

K $\alpha$ radiation $(\lambda=1.5418 \AA$ ). The $U V-v i s$ diffusive reflectance spectrum was performed in a Shimadzu, Mini 1240. The groups on the samples were studied by infrared spectroscopy using a FT-IR Shimadzu- 8400S Spectrometer. 


\section{Result and discussion}

FTIR spectrum of the product has been shown in Fig. 1. The product reveals absorption peaks at $470,532,603,651,665,759 \mathrm{~cm}^{-1}$, which are characteristic peaks for the $\mathrm{Sb}-\mathrm{O}-\mathrm{Sb}$ bands.

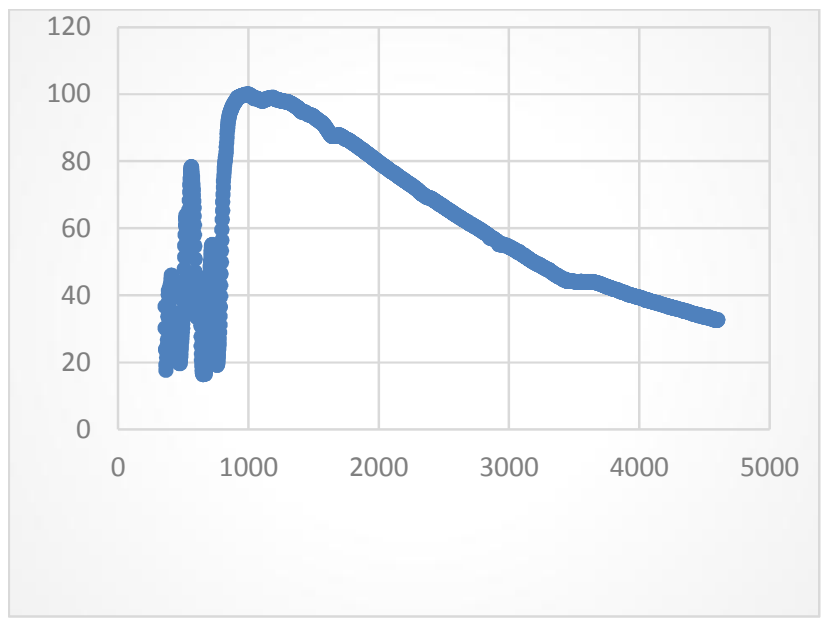

Fig 1. FT-IR spectrum of the product $\left(\alpha-\mathrm{Sb}_{2} \mathrm{O}_{4}\right)$

The XRD pattern as given in Fig. 2 confirms that the product is pure antimony oxide, $\alpha-\mathrm{Sb}_{2} \mathrm{O}_{4}$, with orthorhombic structure (JCPDS Card No. 01-071-0143) [7].

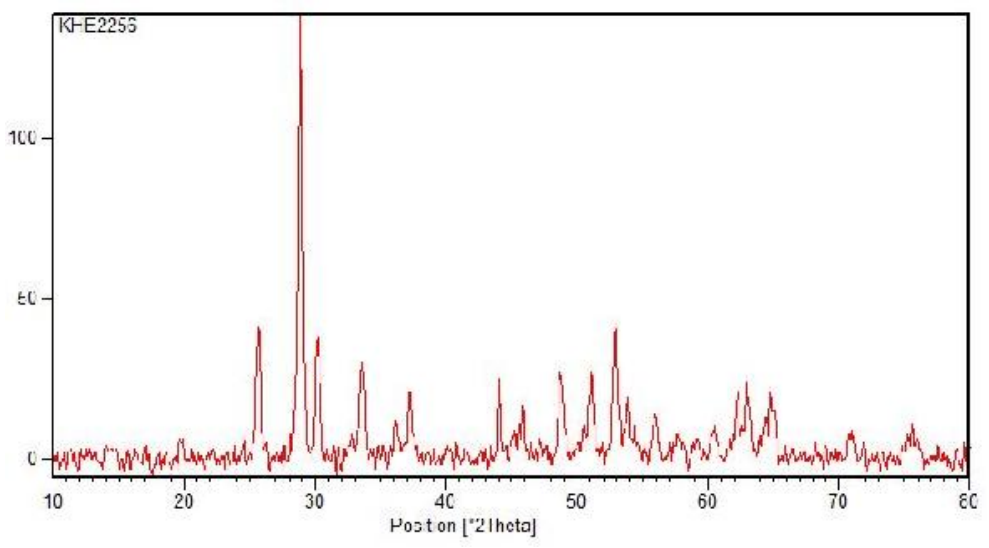

Fig 2. XRD pattern of the product $\left(\alpha-\mathrm{Sb}_{2} \mathrm{O}_{4}\right)$ 
The band gap of $\alpha-\mathrm{Sb}_{2} \mathrm{O}_{4}$ nanostructure is $3.6 \mathrm{eV}$, which was determined by the UVVis spectrum. Fig. 3 indicates the FE-SEM image of antimony oxide, $\alpha-\mathrm{Sb}_{2} \mathrm{O}_{4}$, with leaf-like morphology. The diameter and width of leaf-like nanostructures are 150-200 $\mathrm{nm}$ and 80-100 $\mathrm{nm}$, respectively.

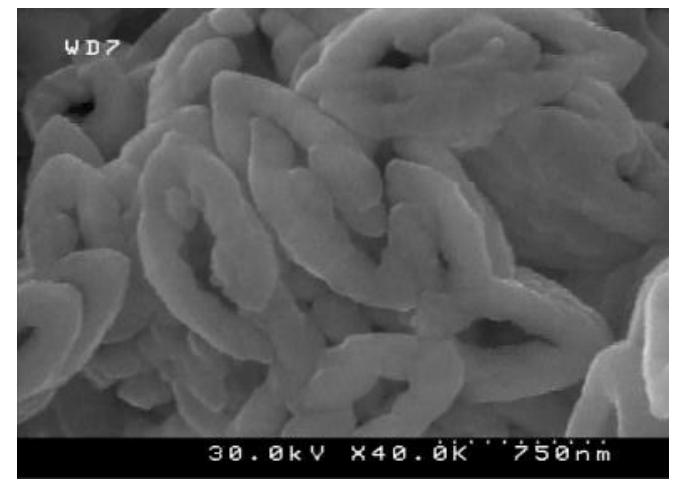

Fig 3. SEM image of leaf like $\alpha-\mathrm{Sb}_{2} \mathrm{O}_{4}$ nanostructure

\section{Conclusion}

We synthesized leaf-like $\alpha-\mathrm{Sb}_{2} \mathrm{O}_{4}$ nanostructure by microwave method for the first time. FT-IR spectrum confirms the formation of the $\alpha-\mathrm{Sb}_{2} \mathrm{O}_{4}$ nanostructures. The product was characterized by using XRD pattern. The FE-SEM image reveals morphology of $\alpha-\mathrm{Sb}_{2} \mathrm{O}_{4}$ nanostructures as leaf-like. The band gap of the leaf-like $\alpha-\mathrm{Sb}_{2} \mathrm{O}_{4}$ nanostructure is determined by $\mathrm{UV}-\mathrm{Vis}$ spectrum, which is $3.6 \mathrm{eV}$.

\section{References}

1. D.W. Zeng, C.S. Xie, B.L. Zhu, W.L. Song, Materials Letters 58 (2004) 312-315.

2. D.W. Zeng, B.L. Zhu, C.S. Xie, W.L. Song, A.H. Wang, Materials Science and Engineering A 366 (2004) 332-337.

3. D.W. Zeng, X. Chen, R. Jiang, C.S. Xie, B.L. Zhu, W.L. Song, Materials Chemistry and Physics 96 (2006) 454-458.

4. Q. Sun, Q. Ren, H. Li, Electrochemistry Communications13 (2011) 1462-1464.

5. B. Ouni, M. Lakhdar, R. Boughalmi, T. Larbi, A. Boukhachem, A. Madani, K. Boubaker, M. Amlouk, Journal of Non-Crystalline Solids 367 (2013) 1-7. 
6. Z. Zhang, L. Guo, W. Wang, Journal of Materials Research 16 (2001) 803-805.

7. A. Jamal, M. Rahman, S. Bahadarkhan, M. Abdullah, M. Faisal, A. Asiri, A. Parwazkhan, K. Akhtar, Journal of Chemical Society of Pakistan 35 (2013) 570.

8. Sh. Liu, B. Wen, W. Jiang, Ch. Liu, W. Ding ,N. Wang, W. Chai, Ceramics International 40 (2014) 15991-15995.

9. D. Orosel, P. Balog, H. Liu, J. Qian, M. Jansen, Journal of Solid State Chemistry 178 (2005) 2602-2607.

10. G. Ren, C. Wang, J. Xia, J. Liu, H. Zhong, Materials Letters 63 (2009) 605-607. 